\title{
A machine learning assisted approach for textile formability assessment and design improvement of composite components
}

\author{
Clemens Zimmerling ${ }^{\mathrm{a}, *}$, Dominik Dörr ${ }^{\mathrm{a}}$, Frank Henning ${ }^{\mathrm{a}, \mathrm{b}}$, Luise Kärger ${ }^{\mathrm{a}}$ \\ ${ }^{\text {a }}$ Karlsruhe Institute of Technology (KIT), Institute of Vehicle System Technology, Karlsruhe, Germany \\ ${ }^{\mathrm{b}}$ Fraunhofer Institute of Chemical Technology (ICT), Pfinztal, Germany
}

Keywords:

A. Fabrics/textiles

C. Computational modelling

C. Statistical properties/methods

E. Forming

Machine learning

\begin{abstract}
A B S T R A C T
Manufacturing continuous fibre reinforced components often involves a forming process of textiles. Process simulations using Finite Element (FE) techniques allow for an accurate virtual formability assessment, but are typically time-consuming, especially for iterative design optimisations. To provide remedy, this work proposes machine-learning (ML) techniques as easy-to-evaluate approximations of FE-forming results. While previous studies focus on adjusting process parameters to achieve manufacturability, this work investigates local geometry variations. Initially, an ML-model is trained on FE-based forming examples in order to relate geometric features to forming results. During component formability assessment, an image-based recognition approach identifies manufacturing-critical regions. Then, the ML-model estimates forming results for each region individually. The validity of local formability assessment for a minimum mutual distance is based on SaintVenant's Principle and is supported by FE-based verification. The overall approach is validated on a complex shaped box-geometry. Moreover, time-efficient exploration of local design alternatives to improve manufacturability is demonstrated.
\end{abstract}

\section{Introduction}

Due to their considerable mechanical properties, continuous fibre reinforced plastics (CoFRP) have drawn wide attention in weight-sensitive industries (e.g. aerospace or automotive). For optimum structural performance, not only the material must be carefully adjusted, e.g. stacking sequence and fibre orientation, but also defect free manufacture needs to be ensured - a challenging engineering task with potentially competing goals.

Manufacturing CoFRP components comprises multiple steps, often involving a forming step of a textile (draping). In textile draping, local defects may significantly reduce the load bearing capacity [1] and therefore, manufacturing effects should be reflected during structural simulations via continuous virtual process chains [2]. Ensuring appropriate formability requires particular attention during component and process design. To achieve defect-free formability, different optimisation approaches have been developed, which concentrate on process parameter adjustment (e.g. gripper forces) for improved forming results [3-5]. However, in many cases manufacturing problems stem from inadequate component design rather than deficient process configuration [6]. Thus, although considerable improvements can be achieved through process optimisation, the potential gains are limited and certainly cannot compensate for an arbitrarily detrimental component design. In consequence, consideration of manufacturing during component design greatly contributes to a lean development and prevents costly redesign loops. Overall, a concurrent structure and process optimisation including geometry variations may be seen as the ultimate goal of CoFRP component design.

This work addresses forming of plain-weave fabrics. A frequently used criterion to assess the draping quality is the in-plane shear angle $\gamma_{12}[5,7,8]$, which mainly forms in doubly-curved regions. Thus, local component curvature limits the manufacturability of the part and requires special attention during component design. Physically motivated simulations by Finite Element (FE) models allow for a detailed description of the forming process. Amongst other things, they pay special attention to accurately modelling the non-linear material behaviour [9]. This is a challenging task, since material testing for model calibration and validation is time- and cost-intensive and, in case of novel or unusual material behaviour, new or enhanced modelling approaches even need to be developed. While FE-techniques promise high physical accuracy, they in turn require profuse numerical expertise for model configuration and considerable computational resources. Especially at early stages of the design process, when many design iterations need to be evaluated, this quickly become laborious and time-consuming. 
Consequently, time-efficient models are a key factor in process and part design strategies for CoFRPs.

Different approaches to time-efficiently account for manufacturing during design have been developed: They range from qualitative design and process selection guidelines [10], via analytical relations for simple shapes [11] and geometry analysis tools, e.g. [12], to advantageous shapes deduced from reverse draping approaches [13]. However, most widespread for rapid forming assessment are purely geometric draping simulations, so called kinematic methods. They are computationally inexpensive, but involve many simplifying assumptions (cf. [14,15]) and disregard the actual physics (e.g. process conditions, friction or material behaviour). Thus, current approaches for time-efficient formability assessment are of limited accuracy.

The advent of Machine Learning (ML) introduces another option for time-efficient process models. ML aims at constructing a numerically inexpensive, phenomenological relationship between input (e.g. process, material and/or geometry parameters) and corresponding output (part quality). Loosely speaking, these models 'learn' the underlying system dynamics from supplied input-output-examples and estimate the outcome of new parameters [16]. Currently, in composite engineering most ML-based applications focus on structural optimisation, e.g. part stiffness or vibration characteristics, e.g. [17-19]. Although, beneficial time-savings during optimisation are reported, e.g. [20], most approaches are component-specific and cannot be reused in other engineering problems [21]. Consequently, developing generalised ML models is an open field of research. Current generalisation approaches for CoFRP focus on material properties, e.g. elastic constants [22,23], but do not consider effects on component scale. To the author's knowledge, our previous works [24-26] are the first studies on component scale which apply ML techniques for textile forming optimisation and forming-related composite design analysis. Results show that current ML-techniques are capable of reproducing results from FE forming simulations.

\subsection{Scope of this work}

This work investigates the idea of using ML-techniques in textile formability assessment of variable geometries to obtain physically accurate predictions while maintaining computational efficiency. The overall aim is to recognise distinct geometric features in a component and make on the corresponding local forming result by an ML-algorithm. The predicitive ML-algorithm learns the forming dynamics from a data base of physics-based FE-draping examples. For preclusion of potential overlay effects, advanced material behaviour effects, e.g. onset of curing/crystallisation or rate-dependent behaviour, are not considered. This allows the isolated study of the algorithm's learning capacity vis-à-vis geometry variations. Feature recognition is performed on an image-based geometry representation. Being a frequent and severe forming challenge, rounded corners serve as an example use-case. For first implementation and general verification of approach validity, this work eliminates corner interaction effects through spatial separation: According to Saint-Venant's Principle (SVP), corner strain fields ought to form independently given a minimum distance. While this work uses fabric forming for approach validation, the overall idea is deemed applicable to other material classes as well (e.g. stitched noncrimp fabrics).

The paper is organised as follows: Section 2 describes the overall methodology of design assessment including a brief introduction of the used ML-technique and the geometry recognition approach. Section 3 presents the FE-forming setup and data processing strategy used for training data generation. Numerical evidence on the validity of independent evaluation of geometric features (Saint-Venant's Principle) is presented in Section 4. Ultimately, the approach is used to time-efficiently predict and improve the manufacturability of a complex shaped box-geometry in Section 5 .

\section{Methodology}

\subsection{General workflow}

Conceptually, this work treats an FE forming simulation as a function $\varphi_{\mathrm{FEM}}: G \mapsto Q$ that maps a set of geometry parameters $G$ to a resulting set of part quality attributes $Q$. Analogous to previous work on draping optimisation, e.g. $[5,7,8]$, the part quality $Q$ is described by the in-plane shear angle $\gamma_{12}$, i.e. $Q=\gamma_{12}$. Since the evaluation of $\varphi_{\mathrm{FEM}}$ is costly and laborious (simulation run), the general aim is to establish an approximative ML-based model function $\mu_{\mathrm{ML}}: G \mapsto Q$ to substitute $\varphi_{\mathrm{FEM}}$ (i.e. ground truth) in a certain target region $G_{\text {target, }}$ i.e. $\mu_{\mathrm{ML}} \approx \varphi_{\mathrm{FEM}} \quad \forall \quad \boldsymbol{g} \in G_{\text {target }} \subset G$.

It may be noted, that $\varphi_{\mathrm{FEM}}$ can be evaluated (i.e. simulation run), but is otherwise unknown ("black-box"). Therefore, classical approximation techniques, e.g. Taylor Series expansion, are inapplicable, since mathematical prerequisites (continuity, differentiability, etc.) cannot be ensured. Even if the prerequisites were satisfied, numerical differentiation rapidly exceeds reasonable computational efforts for higher derivatives in multi-dimensional spaces. Under these circumstances, only data-driven approaches are eligible that approximate $\varphi_{\mathrm{FEM}}$ based on $n$ evaluations - the core task of ML.

Evaluation of the model function $\mu_{\mathrm{ML}}$ is embedded in a workflow as schematically illustrated in Fig. 1. The algorithm accepts a 3D-geometry of interest as an input and identifies geometric features (i.e. corners). Each recognised geometric feature in the component is assigned a set of describing geometry parameters $\boldsymbol{g}_{\boldsymbol{i}}$. All features are collected in a set $\mathscr{C}^{k}=\left\{\mathbf{g}_{1}, \mathbf{g}_{2}, \ldots m, \mathbf{g}_{k}\right\}$. For a valid prediction, the identified parameters $\mathbf{g}_{\boldsymbol{i}} \in \mathscr{C}^{k}$ must lie inside the range of a previously sampled training data set $\mathscr{D}^{n}$ for the model function $\mu_{\mathrm{ML}}$ (i.e. $\forall \mathbf{g}_{\boldsymbol{i}}: \mathbf{g}_{\boldsymbol{i}} \in \operatorname{Conv}\left(\mathscr{D}^{n}\right)$ ), where $\operatorname{Conv}\left(\mathscr{D}^{n}\right)$ denotes the convex hull of $\mathscr{D}^{n}$. Otherwise, $\mathscr{D}^{n}$ needs to be extended by additional samples. For maximum information gain, a design of experiments (DoE) strategy may be used. Ultimately, for each $\boldsymbol{g}_{\boldsymbol{i}}$ in an ML-algorithm $\mu_{\mathrm{ML}}$ previously trained on $\mathscr{D}^{n}$ predicts the prospective forming result (i.e. $\gamma_{12}$ ).

\subsection{Machine learning model}

The model function $\mu_{\mathrm{ML}}$ is preselected from a function class $\mathscr{M}$. A basic requirement of $\mathscr{M}$ is that its functions are highly-flexible, so that they are able to conform to any ground truth. Common function classes are polynomials, support vector machines, decision trees, artificial neural networks and Gaussian Process Regression (GPR) [27]. This work employs GPR, also known as Kriging, as it has shown superior prediction performance in other engineering applications [28-30]. The performance of ML-models is usually quantified by error metrics, which measure how well the model captures the data-inherent dynamics and generalises to unknown input. Common error metrics are e.g. mean square error, coefficient of determination or maximum absolute deviation (outlier analysis). They are usually determined by model validation techniques, such as cross-validation, or are measured on a separate test set [16]. Thereby, error metrics provide guidance on the choice of suitable model functions, possibly complemented by existing knowledge on the problem (e.g. a priori known periodicity). With these metrics, a preceding comparative study of the authors revealed superior predictive capabilities of GPR compared to standard regression approaches, which were found susceptible to outlier-predictions, see [26] for model validation details. Additionally, model training is less expensive compared to e.g. deep neural networks, and in sparse-data-situations GPR is less prone to data-overfitting [31].

GPR is a non-parametric universal approximator based on Bayesian statistics theory [32]. Suppose, a data set $\mathscr{D}^{n}=\left\{\left(\mathbf{g}_{1}, \gamma_{12}^{1}\right), \ldots m,\left(\mathbf{g}_{n}, \gamma_{12}^{n}\right)\right\}$ with $n$ stored observations of input points $\mathbf{g}_{1 \ldots n} \in G$ and corresponding output $\gamma_{12}^{1 \ldots n} \in Q$ has been sampled. GPR then aims at inferring the most likely distribution, that the observations in $\mathscr{D}^{n}$ have been sampled from. To that, between each two points a suitable covariance function $k_{i j}$ 


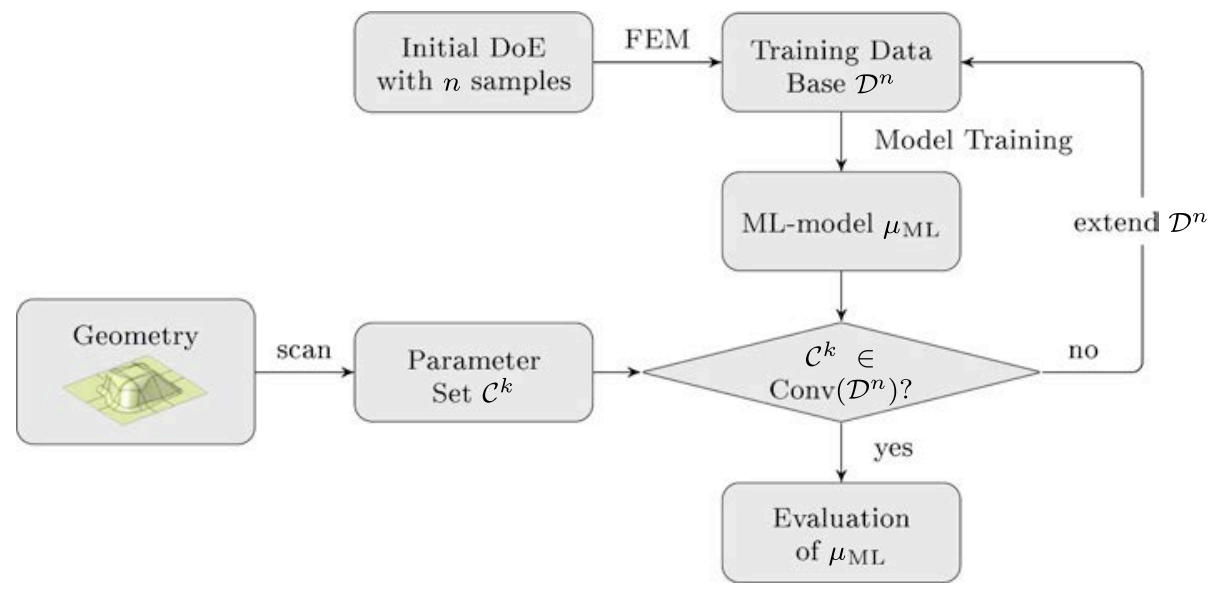

Fig. 1. Schematic workflow of the proposed design evaluation algorithm. After initial training of a model function, geometries of interest are scanned, relevant geometry features are extracted and timeefficiently evaluated by the model function. (For interpretation of the references to colour in this figure legend, the reader is referred to the web version of this article.)

("kernel") is selected. Along with a preselected mean, commonly 0, this fully defines the Gaussian prior distribution. The kernels convey the notion, that close points in the input space $\left(\left|g_{i}-g_{j}\right| \rightarrow 0\right)$ yield alike results and remote points $\left(\left|g_{i}-g_{j}\right| \rightarrow \infty\right)$ do not influence each other. A common choice for $k_{i j}$ is a squared exponential function:

$k\left(x_{i}, x_{j}\right)=k_{i j}=\sigma_{0}^{2} \exp \left(-\frac{1}{2}\left(\frac{\left|\boldsymbol{g}_{i}-\boldsymbol{g}_{j}\right|}{\lambda}\right)^{2}\right)$,

where $\boldsymbol{g}_{i}=\left(g_{1}^{i}, \ldots m, g_{D}^{i}\right)$ are points in the $D$-dimensional input space. The maximum allowable amplitude $\sigma_{0}$ and the length scale $\lambda$ are hyperparameters, which are collected in the vector $\theta=\left(\sigma_{0}, \lambda\right)$. The kernels are aggregated in the symmetric covariance matrix $\boldsymbol{K}$. Using Bayes's theorem, model training is then done by maximising the negative logarithmic likelihood $p(\gamma \mid(\mathbf{g}, \theta))$ :

$\ln (p(\gamma \mid(\mathbf{g}, \theta)))=-\frac{1}{2} \boldsymbol{\gamma}^{T} \boldsymbol{K}^{-1} \boldsymbol{\gamma}-\frac{1}{2} \ln (\operatorname{det}(\boldsymbol{K}))-\frac{n}{2} \ln (2 \pi) \stackrel{!}{\rightarrow} \max$.

Predictions $\hat{\gamma}_{12}$ for a new input point $\hat{\mathbf{g}}$ are possible by evaluation of $\hat{\gamma}_{12}=\hat{\boldsymbol{k}} \boldsymbol{K}^{-\boldsymbol{\gamma}} \boldsymbol{\gamma}$, wherein the vectors $\hat{\boldsymbol{k}}$ and $\boldsymbol{\gamma}$ are defined by $\widehat{\boldsymbol{k}}=\left(k\left(\widehat{\mathbf{g}}, \mathbf{g}_{\mathbf{1}}\right), \ldots m, k\left(\widehat{\mathbf{g}}, \mathbf{g}_{\mathbf{n}}\right)\right)$ and $\boldsymbol{\gamma}=\left(\gamma_{12}^{1}, \ldots m, \gamma_{12}^{n}\right)^{T}$, respectively.

\subsection{Geometry encoding and recognition}

Integral part of the algorithm is the ability to recognise features in a given geometry. From a manufacturing perspective, formed geometries must necessarily be undercut free to allow for collision-free tool closure. Therefore, without loss of information, a bijective projection of the geometry into the tool-plane is tractable, which can be encoded in an image. Besides an advantageous dimensional reduction to a $2 \mathrm{D}$ representation, an image-based representation enables access to numerous processing techniques. Specifically, advanced ML-algorithms

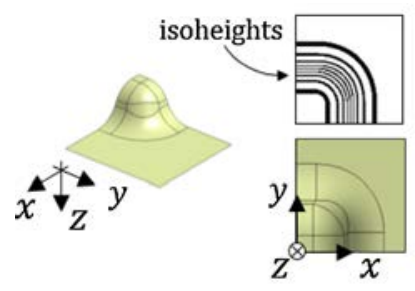

a)

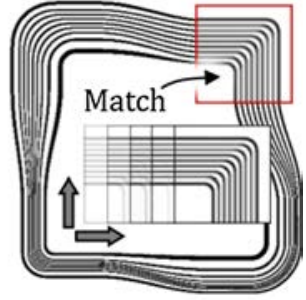

b)
Fig. 2. Visualisation of the geometry encoding scheme in tooling direction $z$ along with the corresponding isoheights-image (a) and the corresponding 2D image-based recognition approach by pattern matching (b). (For interpretation of the references to colour in this figure legend, the reader is referred to the web version of this article.) specialised in computer vision enable object recognition, classification and tracking in images [33]. However, proper configuration of such advanced algorithms is time-expensive. Thus, for first implementation and assessment of the general approach feasibility, a simplified feature recognition technique is applied, namely $2 \mathrm{D}$ normalised cross-correlation [34].

This work uses a topographic map with contour lines of constant elevation (isoheights), cf. Fig. 2a). Beginning from the top, isoheights divide the geometry in user-specified intervals in tool direction $z$, e.g. this study uses $5 \mathrm{~mm}$. Since pixels carry spatial information, image resolution is a critical parameter for information conservation and requires particular attention. If the resolution is too low, processing is fast, but geometry information is not retained and vice versa. In this work, a resolution of $0.25 \mathrm{px} / \mathrm{mm}$ is empirically found to yield a good trade-off for time-efficient but satisfactory recognition confidence $\left(c_{\text {cor }} \geqslant 0.85\right.$, cf. Eq. (3))

The geometric features must be detected irrespective of their position in the component. Consequently, translation invariant feature recognition is required. It may be noted, that rotation invariance is not aspired in this work since forming results strongly depend on the relative angle between fabric and geometry feature due to anisotropic material behaviour. Additionally, scale invariance is not pursued since pixels carry spatial information and thus, images must not be scaled.

Inspired by image processing in neural networks, convolving templates across a target image (investigated geometry) ensures translation invariance. The convolution operation may be seen as sliding a template over the target image until a match (i.e. high correlation) is observed. Since pixels are discretised values, a discrete convolution operation is performed yielding the normalised 2D cross-correlation [34]:

$c_{\text {cor }}(u, v)=\frac{\sum_{x} \sum_{y}\left[T(x, y)-\bar{T}_{x y}\right][\vartheta(x-u, y-v)-\bar{\vartheta}]}{\sqrt{\sum_{x} \sum_{y}\left(T(x, y)-\bar{T}_{x y}\right)^{2} \cdot \sum_{x} \sum_{y}(\vartheta(x-u, y-v)-\bar{\vartheta})^{2}}}$

wherein $T(x, y)$ is the target image, $\bar{T}_{x y}$ the average of $T(x, y)$ under the template $\vartheta$ positioned at $(u, v)$. Summation range for $x$ and $y$ is the region under $\vartheta$. For increased recognition robustness, in this work, the isoheights are additionally thickened by two pixels to allow for minor mismatches between template and target. The templates stem from a large repository with $n_{\mathrm{tpl}}=1372$ geometry samples with known parameters $\mathbf{g}$. The procedure is visualised in Fig. 2 b). The according geometry parameters are then extracted from a lookup table and parsed to the ML-model $\mu_{\mathrm{ML}}$ for evaluation. 
Table 1

Geometry parameterisation scheme, considered parameter range and geometry examples for visualisation.

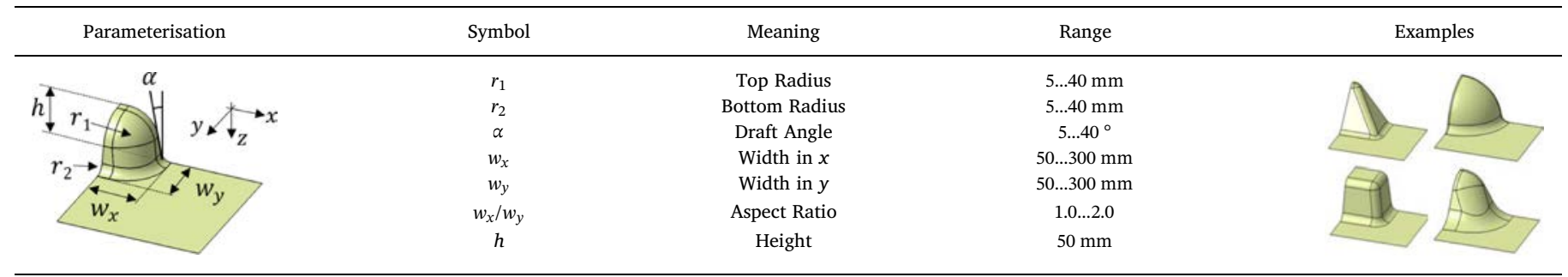

\section{Training data generation from forming simulations}

\subsection{Preprocessing}

For automated geometry generation, a parametric CAD-model is used. The parameterisation scheme and range (cf. Table 1) is inspired by $[35,28]$ and aims at a wide coverage of possible corner geometries. In future applications, additional schemes may be used to enhance the overall scope of geometric features to a holistic 'draping catalogue'.

For maximum information gain during exploration of the geometry parameter space G, Latin Hypercube Sampling (LHS) [36] is used. Amongst others, it is a popular space-filling sampling plan for deterministic computer experiments. It strives for a uniform distribution of sample points in the geometry parameter space $\left(r_{1}, r_{2}, \alpha, w_{x}, w_{y}\right)=\mathbf{g} \in G$, while avoiding recurrences of parameter levels. Since extrapolations are generally inadvisable, additional samples at the boundary of $G$ are drawn using Box-Behnken- and a full factorial design, as suggested in [37]. Doubly-symmetric tool surfaces are deduced from the corner geometry samples $\mathbf{g}_{i} \in G$. To build the data base $D^{n}$, a significant number of simulations is required and thus only socalled macroscopic forming simulations are computationally practicable. They treat each ply within the stack as one continuum with homogenised mechanical properties and have reached a remarkable level of physical accuracy even for complex textile architectures [38]. Subsequently, FE-draping simulations are set up within an in-house developed forming simulation framework based on the commercial FEcode ABAQus[39,9]. This framework conveniently enables a fully automatised pre- and postprocessing of draping simulations. Also, it comprises a subroutine library for several constitutive models describing intra- and inter-ply behaviour. A single ply fabric is formed in one stroke within $1 \mathrm{~s}$. The fabric is always of rectangular shape. To account for the decoupled membrane and bending behaviour, the layer consists of superposed membrane (M3D3) and conventional shell elements (S3R). Thickness-compaction is neglected, i.e. a constant layer thickness is assumed. A visualisation of an example simulation setup is given in Fig. 3.

In this work, a hyperelastic constitutive equation for the non-linear membrane behaviour is used with a constant in-plane shear modulus $G_{12}=1 \mathrm{MPa}$ and a longitudinal stiffness in warp and weft direction $E_{1}=E_{2}=1000 \mathrm{MPa}$. The material model enables a covariant description of material stiffnesses at large strains, so that its behaviour in non-orthogonal, fibre-parallel frames can be correctly described, see e.g. $[9,39,40]$ for modelling details and experimental validation. For reference, Fig. 3c) shows the results of a virtual bias extension test of the material. The curves qualitatively agree with experimental findings for fabric reinforced thermoplastic materials at low deformation rates and moderate temperatures $[41,42]$. The selected material configuration facilitates sufficiently large membrane section forces as required for validation of Saint-Venant's principle (cf. Section 4). A Coulomb friction coefficient of $\eta=0.3$ models tool-ply contact. Isothermal process conditions as well as spatially and time-wise constant material properties are assumed, i.e. rate-dependencies and additional effects from curing or crystallisation for respective material classes are not considered. While a detailed experimental validation is beyond the scope of this work, the overall forming behaviour appears plausible compared to aforementioned experimental findings. Owing to the methodological nature of this study, material and process parameters may be perceived as generic and do not aspire comprehensive modelling of a particular material. Altogether, the model is configured so as to allow for an isolated study of the algorithm's learning capacity towards geometry variations.

The in-plane shear-angle $\gamma_{12}$ quantifies the draping quality. Typically, excessive shear deformations increase the likelihood of unwanted defects, such as wrinkling, textile folding or poor resin infiltration due to fibre compaction. Apart from a deficient visual appearance, these defects substantially compromise the load bearing capacity and may lead to part reject [1]. The actual formation of defects is a complex interaction of in-plane stresses, in-plane shear stiffness and out-of-plane-bending stiffness [43]. Thus, for simplicity, this work assumes an empirical limit shear angle $\gamma_{12}^{\lim }=55^{\circ}$ to distinguish defective from defect-free forming results.

\subsection{Postprocessing}

As local instead of global forming results are of interest, postprocessing concentrates on evaluating the shear angles at individual corners. Around each corner's apex, a rectangular evaluation region is defined by $l_{\text {out }}=\frac{1}{\cos (\alpha)} h+r_{1}+r_{2}$ and $l_{\text {in }}=r_{1}$ and projected onto the undeformed fabric, as presented in Fig. 4a). Elements inside the projection are collected in an evaluation set. The region boundaries are not
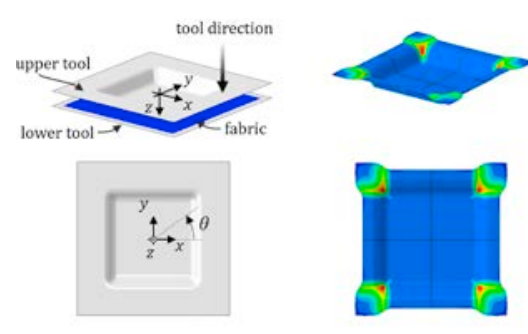

a)

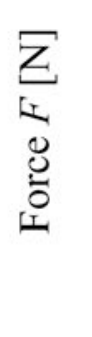

b)

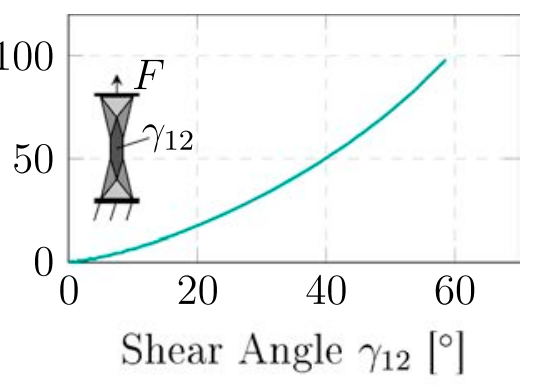

c)

Fig. 3. Visualisation of an exemplary draping simulation setup (a), a qualitative plot of the absolute shear angle distribution after forming (b), nonlinear in-plane shear curve obtained in a virtual bias extension test (c). (For interpretation of the references to colour in this figure legend, the reader is referred to the web version of this article.) 


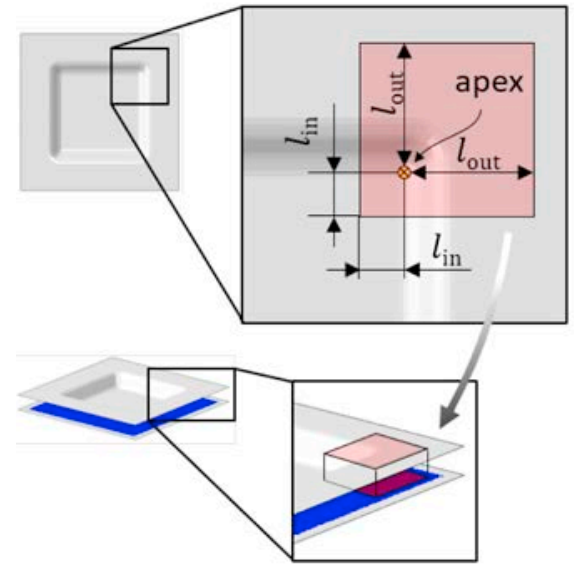

a)
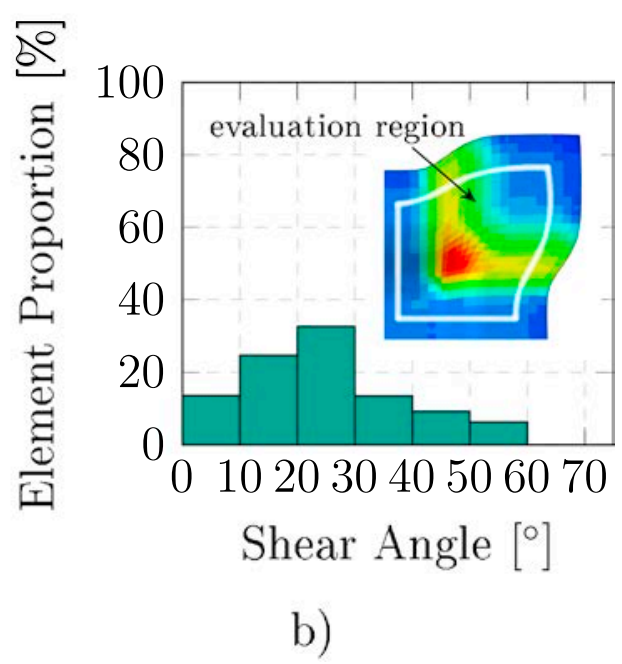

b)
Fig. 4. Definition of evaluation region and projection scheme onto the undeformed fabric (a). Histogram of the shear angle distribution after forming of an exemplary corner geometry along with a qualitative plot of the forming result for visualisation (b). (For interpretation of the references to colour in this figure legend, the reader is referred to the web version of this article.) fix, but depend on the corner parameters, which ensures that the evaluation region always encloses the main deformations independent of the current geometry parameter setting. The shear angles within the evaluation set are collected in a vector $\gamma_{12}=\left(\gamma_{12}^{1}, \ldots m\right)$, where $\gamma_{12}^{i}$ is the shear angle of the $i$-th element in the set. The entries of $\gamma_{12}$ are sorted into a histogram with bins of $10^{\circ}$. An example histogram along with the according evaluation set can be seen in Fig. 4b). As they pose no manufacturing relevance but only distort the histogram, shear angles below $5^{\circ}$ are discarded. From the histogram, statistical metrics are computed, which give a lower dimensional representation of the distribution. Specifically, the maximum shear angle $\gamma_{12}^{\max }$, average shear angle $\gamma_{12}^{\text {avg }}$ and corresponding standard deviation $\gamma_{12}^{\text {std }}$ are used. Higher order statistical moments, e.g. skewness and kurtosis, are also conceptually usable, but being less intuitive they are not considered in this work.

The procedure is repeated for each geometry parameter set $\mathbf{g}_{i}$ and ultimately, the metrics of all training geometries are aggregated in a training data base, e.g. $\mathscr{D}^{n}=\left\{\left(\mathbf{g}_{i}, \gamma_{12_{i}}^{\max }, \gamma_{12_{i}}^{\mathrm{avg}}, \gamma_{12_{i}}^{\mathrm{std}}\right)\right\}, i=1 \ldots n$, and training of $\mu_{\mathrm{ML}}$ is performed according to Eq. (2). In total, $n=81$ samples $\left(n_{\text {train }}=71\right.$ training and $n_{\text {val }}=10$ validation samples) per fibre orientation are found a sufficient data base for the considered corner geometries in this work, cf. [26] for model validation details.

\section{Saint-Venant's principle in fabric forming}

This work assumes, that strain fields of geometric features form independently of each other given a minimum mutual distance (SaintVenant's Principle, SVP). In the following, theoretical background and numerical evidence is provided for justification of this assumption.

One formulation of SVP states, that the order of magnitude $m(\boldsymbol{x})$ of a strain component at a position of interest $\boldsymbol{x}$ in a loaded body is confined by

$m(\boldsymbol{x})=\mathcal{O}\left(\left(\frac{\epsilon}{d(\boldsymbol{x})}\right)^{p}\right)$

if $\epsilon<d(\boldsymbol{x})$ [44]. Therein, $\epsilon$ is the diameter of a conceptual surface $\mathscr{S}$ enveloping the region with the cause of strain (i.e. double-curvature in fabric forming). The variable $d(\boldsymbol{x})$ denotes the distance between $\boldsymbol{x}$ and the region's centre and $p$ is a load case dependent decay exponent, with $p \geqslant 0$ in general. In the following, $d$ is used for $d(\boldsymbol{x})$ for brevity. Clearly, with increasing distance (i.e. $\epsilon / d \rightarrow 0$ ) or higher values for $p, m(\boldsymbol{x})$ decreases and thus, strain fields localise in the vicinity of their cause. The value of $p$ depends on the resulting loads transferred across $\mathscr{S}$, while in general, vanishing resultant loads yield higher values of $p$. In other words, the strain state at $\boldsymbol{x}$ ought to remain nearly unaffected of other strain fields in the body given either a sufficient distance or vanishing resultant loads.

\subsection{Phenomenological study}

A parametric study is used to validate the hypothesis of strain field independence. Six fibre orientations $\Theta$ between $\Theta=0^{\circ}$ and $\Theta=45^{\circ}$ are draped onto box-geometries with constant corner geometries while stepwise increasing the distance $d$ between the corners. Fig. 5 illustrates the procedure exemplarily for a pyramid corner with $\mathbf{g}_{\mathrm{pyr}}=\left(r_{1}, r_{2}, \alpha, w_{x}, w_{y}\right)=\left(5 \mathrm{~mm}, 5 \mathrm{~mm}, 40^{\circ}, 75 \mathrm{~mm}, 75 \mathrm{~mm}\right) \quad$ and $\Theta=22.5^{\circ}$.

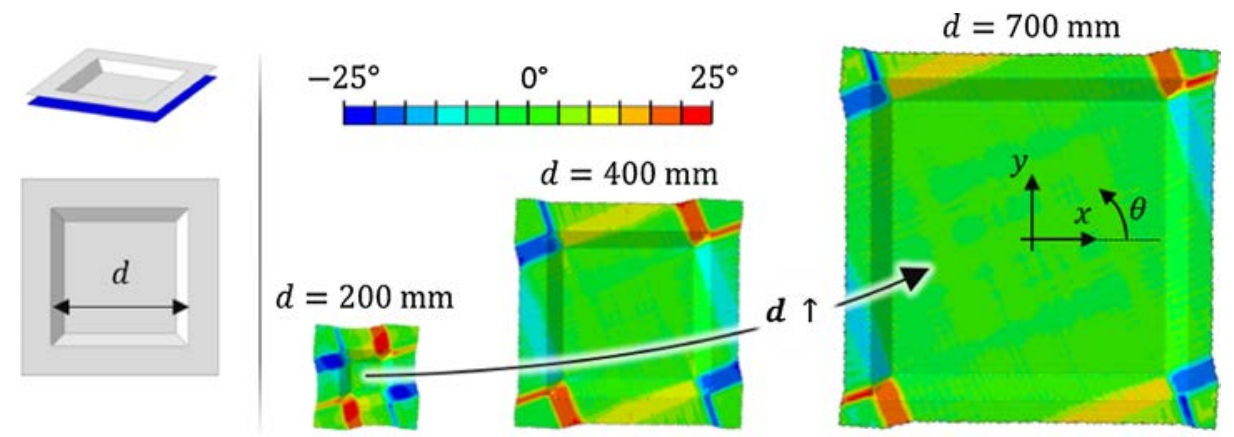

Fig. 5. Setup of the parametric study exemplarily shown for $\Theta=22.5^{\circ}$ and the pyramid corner geometry. The distance $d$ between two corners is stepwise increased while tracking the shear angle statistics. The corner geometries remain constant. (For interpretation of the references to colour in this figure legend, the reader is referred to the web version of this article.) 


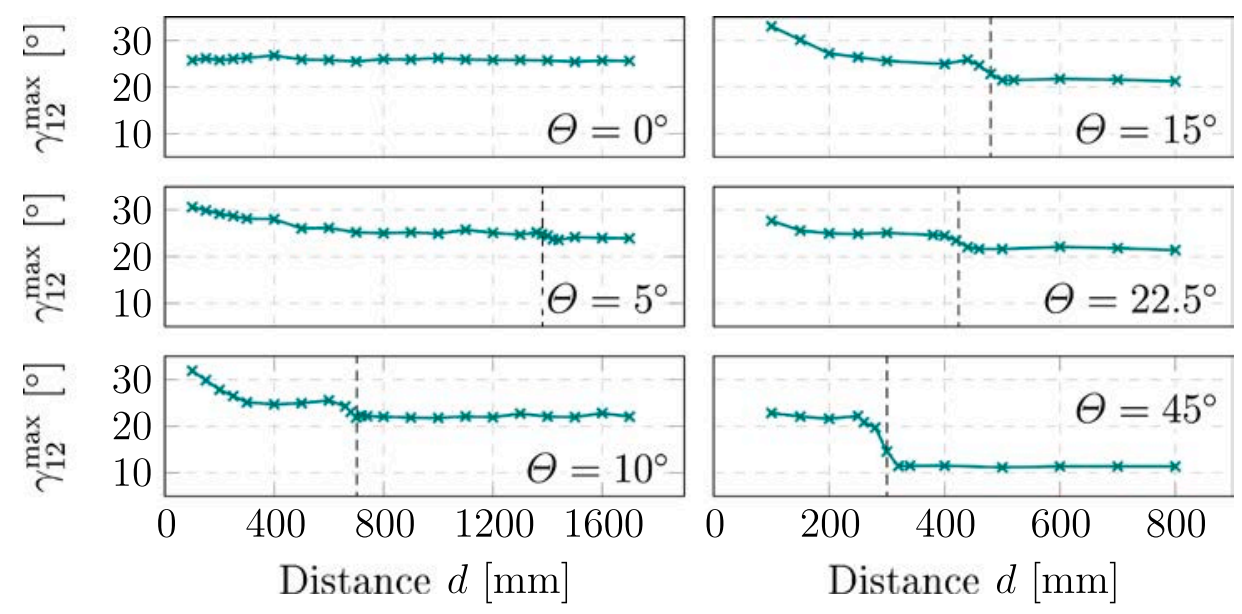

Fig. 6. Evolution of $\gamma_{12}^{\max }$ with the distance $d$ between two pyramid corners. With growing $d, \gamma_{12}^{\max }$ approaches a constant value for each fibre direction $\Theta$, implying strain field independence. Dashed vertical lines mark the predicted minimum distance $d_{\min }$ according to Eq. (5). (For interpretation of the references to colour in this figure legend, the reader is referred to the web version of this article.)

The evolution of shear angles with $d$ is tracked and plotted for visual interpretation (Fig. 6). As it bears most graphicness, the evolution of $\gamma_{12}^{\max }$ is used for discussion. The graphs show three main characteristics: First, starting from a maximum value, $\gamma_{12}^{\max }$ decreases and approaches a minimum value in an asymptotic manner. Secondly, the difference between initial and final values depends on $\Theta$ and is lowest when the fibres are oriented perpendicular to the blank perimeter (i.e. $\Theta=0^{\circ}$ ). Thirdly, each graph with $\Theta \neq 0^{\circ}$ poses a significant drop (dashed vertical lines). Beyond these drops $\gamma_{12}^{\max }$ is approximately constant, which implies mutual independence of corner strain fields. Analogous results are obtained for three other corner geometries.

\subsection{Mechanical justification}

The mutual independence of forming results can be justified by SVP. Consider again the pyramid corner $\mathbf{g}_{\text {pyr }}$ for the doubly-symmetric case $\Theta=45^{\circ}$ with employed symmetry conditions at the boundary (Fig. 7a)). Since only a 2D-shell rather than 3D-body is considered, the enveloping surface $\mathscr{S}$ degenerates to a line $\mathscr{L}$ at the boundary. As can be seen from the qualitative plots, distinct shear bands form along warp and weft direction (Fig. 7b)). Depending on $d$, they end either in $\mathscr{L}$ for small $d$ or in the free edge when $d$ increases. Since shear deformations inevitably cause shear stresses, reaction forces and moments across $\mathscr{L}$ can be extracted. The selected material properties (cf. Section 3.1) facilitate sufficiently large forces and moments for unambiguous separation of intra-ply forces from material strains and inter-ply forces from e.g. friction. Fig. 7 shows their evolution with $d$ by means of the average absolute force per unit length across $\mathscr{L}$ in normal and tangential direction, i.e. $f_{\mathrm{n}, \mathrm{t}}^{\text {avg }}=\operatorname{mean}\left(\left|f_{\mathrm{n}, \mathrm{t}}\right| \mathscr{L}\right)$. Also the according mean absolute line moment $m_{\mathrm{z}}^{\text {avg }}$ about the $z$-axis with respect to the origin $O$ is given, i.e. $m_{\mathrm{z}}^{\text {avg }}=\operatorname{mean}\left(\left|f_{\mathrm{n}} x\right|_{\mathscr{L}}+\left|f_{\mathrm{n}} y\right|_{\mathscr{L}}\right)$. Note, that the forces and moments are normalised to account for continuous growth of boundary length with $d$.

The line forces $f_{\mathrm{n}, \mathrm{t}}^{\text {avg }}$ decrease with $d$ and pose a minimum at about $d=300 \mathrm{~mm}$. The line moment $m_{\mathrm{z}}^{\text {avg }}$ is initially approximately constant but then also drops precipitously at $d=300 \mathrm{~mm}$. In consequence, virtually no resulting load from the strain field is transferred across the boundary for $d>300 \mathrm{~mm}$. The minor growth of $f_{\mathrm{n}, \mathrm{t}}^{\text {avg }}$ stems from
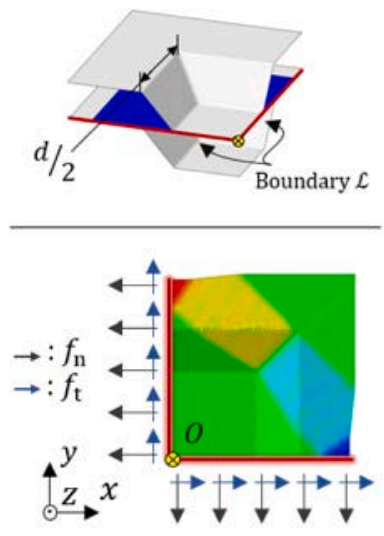

a)

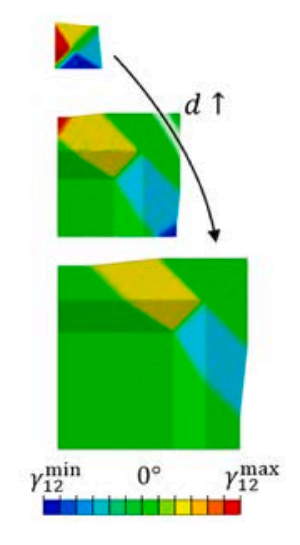

b)
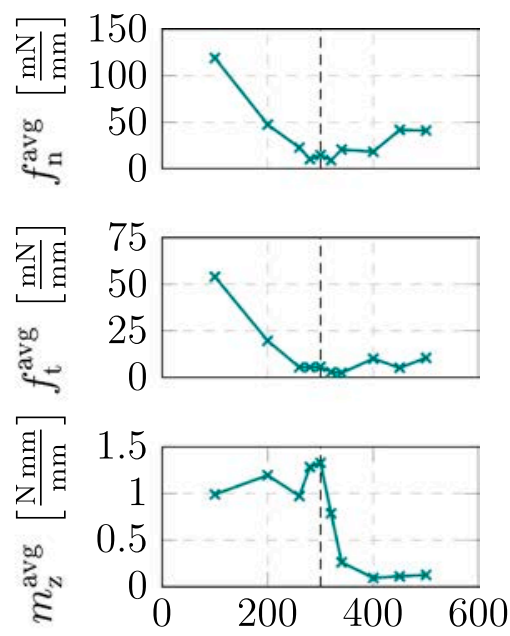

Distance $d[\mathrm{~mm}]$

c)

Fig. 7. Schematic visualisation of the simulation setup and extraction scheme of loads transmitted through the boundary for $\Theta=45^{\circ}$ and $\mathbf{g}=\mathbf{g}_{\text {pyr }}$ (a). Visualisation of shear bands movement from the boundary to the free edge with growing $d$ (b). Plot of the evolution of transmitted loads with $d$ (c). (For interpretation of the references to colour in this figure legend, the reader is referred to the web version of this article.) 
increasing inter-ply frictional forces between tool and ply due to greater contact area rather than from material strains. This approximate absence of transmitted forces satisfies the special conditions of an astatic equilibrium, i.e. resulting tractions and moments are in equilibrium and remain in equilibrium when rotated about an arbitrary angle. For an astatic equilibrium, $p \geqslant 4$ prevails in Eq. (4) [44]. Thus, SVP predicts a strong localisation effect for $d>d_{\mathrm{SVP}}=300 \mathrm{~mm}$. Compared to the corresponding shear angle evolution (Fig. 6, bottom right), precisely at $d=d_{\mathrm{SVP}}=300 \mathrm{~mm}$ (dashed vertical line) a distinct drop of $\gamma_{12}^{\max }$ to a constant value is apparent (i.e. strain field independence) as predicted by SVP. Therefore, SVP is applicable and justifies an individual assessment of forming results.

\subsection{Minimum distance for independence}

Visual comparison of $d<300 \mathrm{~mm}$ and $d>300 \mathrm{~mm}$ (cf. Fig. 7b) and (c)) indicates, that shear bands need to end on the free edge for full independence. Thus, pure geometric considerations enable the determination of an independence criterion of two neighbouring corners: Conceptual straight lines through the double curvature's apexes in warp and weft direction must coincide outside the fabric. For graphicness, shear angles after forming are plotted onto the initial, undeformed configuration (cf. Fig. 8).

As an example, a rectangular fabric cut is considered. With the distance $l$ of the corners's apexes to the fabric's edge and the fibre orientations $\Theta$, the minimum required distance for independence is found to be

$d_{\min }=l \cdot\left(\tan (\Theta)+\frac{1}{\tan (\Theta)}\right)$.

Other formulae may be derived for different sheet geometries. Note, that $d_{\min }$ is independent of the actual corner geometry and depends only on fibre direction $\Theta$ and the apex's distance to the fabric edge $l$. For validation of Eq. (5), the predicted independence distances $d_{\min }(\Theta)$ are also included in Figs. 6 and 7 as dashed vertical lines. Since they coincide with the observed drops of $\gamma_{12}^{\max }$ to a constant level and the minimum of $f_{\mathrm{n}}^{\text {avg }}$ and $m_{\mathrm{z}}^{\text {avg }}$, respectively, they validate the independence criterion and the validity of SVP. Since the drops diminish gradually for $\Theta \rightarrow 0^{\circ}, d_{\text {min }}\left(\Theta \leqslant 10^{\circ}\right)=d_{\text {min }}\left(\Theta=10^{\circ}\right)$ may be used to prevent $d_{\text {min }} \rightarrow \infty$. In the special case of $\Theta=0^{\circ}$, immediate independence is observed, thus $d_{\min }\left(\Theta=0^{\circ}\right)=0 \mathrm{~mm}$ complements Eq. (5).

\section{Application example}

In the following, the overall approach is exemplarily applied to rapidly assess and improve manufacturability of a generic, complex shaped box-geometry, whose corners fall into the parameterisation scheme delineated above. Two fibre orientations are considered $\left(\Theta=0^{\circ}\right.$ and $\Theta=45^{\circ}$ ). The distance $d$ between the corners reflects the minimum

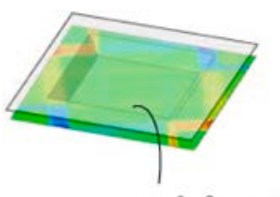

undeformed

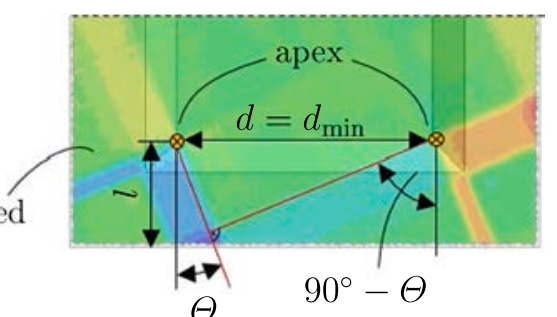

$\Theta$

Fig. 8. Exemplary determination of the minimum required distance $d_{\min }$ for independence of two doubly-curved regions aligned to the edges of a rectangular fabric cut. The plot shows the limit case of beginning independence (i.e. $d=d_{\min }$ ). As $d$ increases, the shear bands separate further, as $d$ decreases, the shear bands move together and shear strains interact. (For interpretation of the references to colour in this figure legend, the reader is referred to the web version of this article.)

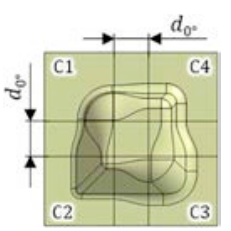

\begin{tabular}{cccccc} 
Corner & $\frac{r_{1}}{m m}$ & $\frac{r_{2}}{m m}$ & $\frac{\alpha}{\circ}$ & $\frac{w}{m m}$ & $\frac{l}{m m}$ \\
\hline C1 & 40 & 10 & 10 & 35 & 35 \\
C2 & 10 & 10 & 40 & 15 & 15 \\
C3 & 40 & 20 & 35 & 25 & 25 \\
C4 & 15 & 15 & 15 & 30 & 30
\end{tabular}

Fig. 9. Visualisation of the box-geometry (left) and the governing geometry parameters for each corner to create the example box-geometry considered in this work. (For interpretation of the references to colour in this figure legend, the reader is referred to the web version of this article.)

required distance for independence $d_{0^{\circ}}=50 \mathrm{~mm}>d_{\min }\left(\Theta=0^{\circ}\right)$ and $d_{45^{\circ}}=175 \mathrm{~mm} \approx d_{\min }\left(\Theta=45^{\circ}\right)=170 \mathrm{~mm}$ for $l=85 \mathrm{~mm}$. (See Fig. 9).

\subsection{Geometry recognition}

Image-based geometry parameter extraction is performed following the approach described in Section 2.3. Converting 3D-geometries to a $2 \mathrm{D}$ isoheight image and convolving the templates yields the location of the corners. Convolving one template takes about $240 \mu$ s on a nVidia $1050 \mathrm{Ti}^{\mathrm{TM}}$-GPU with a total recognition time for $n_{\mathrm{tpl}}=1372$ geometry templates of about $5 \mathrm{~min}$. The best matches are plotted into the target image along with the correlation coefficient $c_{\text {cor }}$. An example of the output-image is given in Fig. 10. The correlation coefficient $c_{\text {cor }}$ is inserted as well for each corner, showing a recognition confidence of $c_{\text {cor }} \geqslant 0.91$ for the considered corners. Empirically, $c_{\text {cor }} \geqslant 0.85$ is found sufficient, to ensure that the mismatch between identified and actual parameters is less than $1.5 \mathrm{~mm}$ and $1^{\circ}$, respectively.

Upon closer examination, minor mismatches between target and template can be observed, e.g. in the lower left corner with $c_{\text {cor }}=0.912$. This occurs for two reasons: First the non-continuous discretisation to pixels introduces some error, but more importantly, the geometries of template and target do not match exactly, but are just very similar to each other. Although great care was taken to provide a sufficiently densely sampled template repository (1372 templates), an exact match for arbitrary geometry parameters is rarely achieved. However, the obtained results hint general validity of image-based recognition.

\subsection{Model evaluation}

The extracted parameters for each corner are parsed to the previously trained model function $\mu_{\mathrm{ML}}$ for evaluation of the shear angle statistics $\left(\gamma_{12}^{\max }, \gamma_{12}^{\text {avg }}\right.$ and $\left.\gamma_{12}^{\text {std }}\right)$. Two fibre orientations are considered

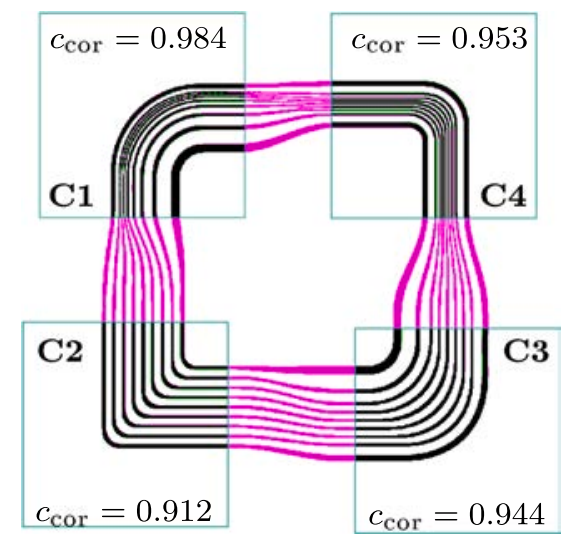

Fig. 10. Visualisation of the recognised geometry features for the considered box-geometry through template matching. Isoheights in identified regions are plotted in black and framed, while non-matching pixels are marked pink. (For interpretation of the references to colour in this figure legend, the reader is referred to the web version of this article.) 


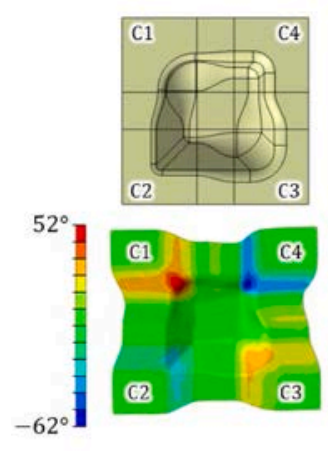

a)

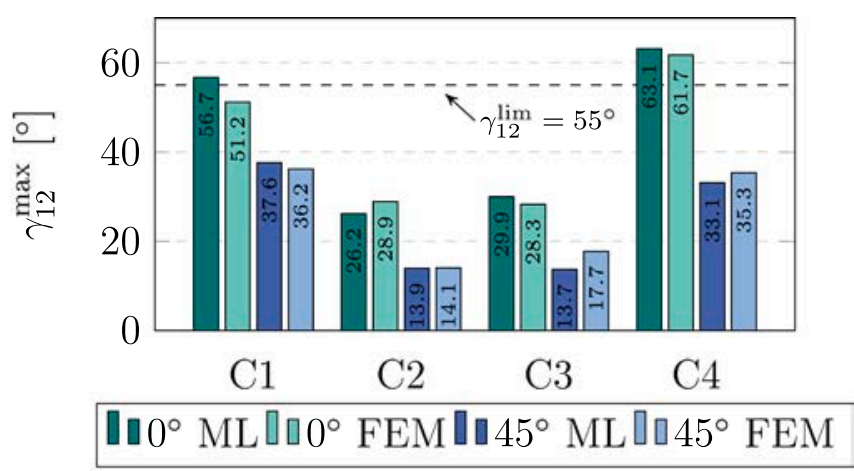

b)

Fig. 11. Visualisation of the Box-geometry and FE-forming result for $\Theta=0^{\circ}$ (a). Comparison of $\gamma_{12}^{\max }$ for each corner as predicted by the ML-model and an FESimulation (b). (For interpretation of the references to colour in this figure legend, the reader is referred to the web version of this article.)

Table 2

Mean and Standard Deviation of the shear angle distribution $\gamma_{12}^{\text {avg }}$ and $\gamma_{12}^{\text {std }}$, respectively, as predicted by the ML-model and an according FE-draping simulation for the considered example box-geometry.

\begin{tabular}{|c|c|c|c|c|c|c|c|c|c|}
\hline & & \multicolumn{2}{|c|}{$\mathrm{C} 1$} & \multicolumn{2}{|c|}{$\mathrm{C} 2$} & \multicolumn{2}{|c|}{ C3 } & \multicolumn{2}{|c|}{ C4 } \\
\hline & & $\gamma_{12}^{\mathrm{avg}}$ & $\gamma_{12}^{\mathrm{std}}$ & $\gamma_{12}^{\text {avg }}$ & $\gamma_{12}^{\mathrm{std}}$ & $\gamma_{12}^{\text {avg }}$ & $\gamma_{12}^{\mathrm{std}}$ & $\gamma_{12}^{\text {avg }}$ & $\gamma_{12}^{\mathrm{std}}$ \\
\hline \multirow[t]{2}{*}{$0^{\circ}$} & ML & 24.9 & 13.7 & 21.3 & 6.8 & 19.2 & 8.0 & 33.2 & 16.4 \\
\hline & FEM & 22.0 & 10.8 & 19.2 & 7.4 & 15.2 & 6.9 & 21.4 & 12.4 \\
\hline \multirow[b]{2}{*}{$45^{\circ}$} & ML & 20.0 & 11.1 & 13.3 & 3.4 & 13.2 & 4.6 & 22.7 & 10.2 \\
\hline & FEM & 22.3 & 10.0 & 13.0 & 4.0 & 12.3 & 4.6 & 22.2 & 10.8 \\
\hline
\end{tabular}

$\left(\Theta=0^{\circ}\right.$ and $\left.\Theta=45^{\circ}\right)$. For validation, corresponding FE draping simulations are performed using the forming framework described in Section 3 (cf. Fig. 11a) for an example plot). Bearing most manufacturing relevance, the ML-prediction and FE-result for $\gamma_{12}^{\max }$ are visually compared in Fig. 11b). Additionally, $\gamma_{12}^{\text {avg }}$ and $\gamma_{12}^{\text {std }}$ are given in Table 2 for completeness. Overall, a good agreement is observed.

The maximum absolute error between FE- and ML-prediction of $\gamma_{12}^{\max }$ is $\approx 5.2^{\circ}(\approx 10 \%)$, while all other differences stay below $3^{\circ}$. Similar good agreement is found for $\gamma_{12}^{\text {aag }}$ and $\gamma_{12}^{\text {std }}$, except for one outlier at corner C4. Upon closer inspection, this particular parameter combination lies in a region, which has only sparsely been sampled, leading to higher predictive uncertainties. Adjusting sampling control parameters for a more uniform sample distribution or a higher number of samples may alleviate this.

\subsection{Design improvement}

According to the ML-prediction in Fig. 11, forming of corner C1 and corner C4 exceeds the limit shear angle $\gamma_{12}^{\lim }=55^{\circ}$ (dashed horizontal line) for the fibre orientation $\Theta=0^{\circ}$, while $45^{\circ}$-plies stay below. Consequently, the current part design does not facilitate manufacture of $0^{\circ}$-plies and requires a redesign.

To explore and visualise the effect of different corner designs, the ML-model $\mu_{\mathrm{ML}}$ can beneficially be employed: In a sensitivity study, draft angle $\alpha$ and fillet radius $r_{2}$ (cf. Table 1) show the highest impact on $\gamma_{12}^{\max }$ and are thus selected for the redesign. Fig. 12 shows an according plot. The red line indicates the locus of designs yielding $\gamma_{12}^{\lim }$ after forming. Designs 'left' of the red line (i.e. $\gamma_{12}^{\max }>\gamma_{12}^{\lim }$ ) are deemed nonmanufacturable and vice versa. This visualisation of $\mu_{\mathrm{ML}}$ facilitates component design: From the 'manufacturable' design zone, the designer can pick any parameters that suit best to other design requirements (e.g. packaging, functional requirements).

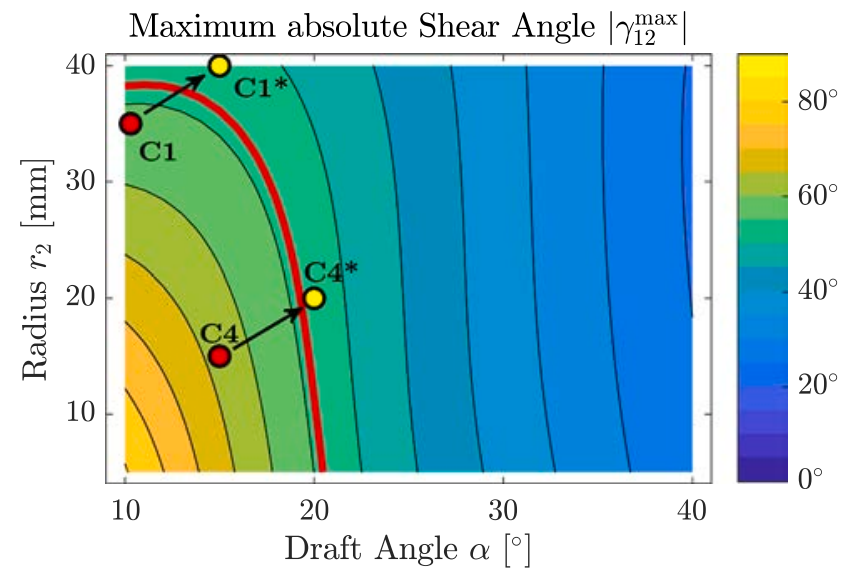

Fig. 12. Corner design map for visual manufacturability assessment. The red line is the locus of $\gamma_{12}^{\max }=\gamma_{12}^{\lim }=55^{\circ}$ and separates manufacturable from nonmanufacturable designs. Markers indicate the location of corner C1 and C4 before (red) and after redesign (yellow). (For interpretation of the references to colour in this figure legend, the reader is referred to the web version of this article.)

Also the current designs of Corner C1 and C4 are marked in Fig. 12. They lie inside the non-manufacturable zone and require thus an adjustment to achieve manufacturability. The successful reduction of $\gamma_{12}^{\max }$ below $\gamma_{12}^{\lim }$ is presented in Fig. 13, along with an FE-simulation for reference. The asterisks denote a redesigned corner. Again, ML-model prediction and $\mathrm{FE}$-simulation match well, with a maximum difference of $\approx 3^{\circ}$ for $\mathrm{C}^{*} *$ and $\mathrm{C} 4 *$. For completeness, the result of $\mu_{\mathrm{ML}}$ and FEresult for $\Theta=45^{\circ}$ before and after redesign are also given, and preservation of manufacturability during redesign is observed, i.e. $\gamma_{12}^{\max }\left(\Theta=45^{\circ}\right)<\gamma_{12}^{\lim }$ for all corners. Consequently, the trained MLmodel function $\mu_{\mathrm{ML}}$ has successfully been applied to efficiently assess manufacturability and facilitated redesigns of individual geometric features.

Finally, an overall reduction of numerical effort is observed. For instance, $n_{\text {eval }}=1116$ function evaluations generate the corner design map (Fig. 12). The ML-model only requires training and validation FEsamples, in this case a total of $n=81$ simulations. Thus, the simulation effort reduces to $n / n_{\text {eval }}=81 / 1116 \approx 7.2 \%$ compared to an entirely FEbased computation. Assuming the model training effort to be small compared to the simulation effort, the numerical break-even-point is then governed by the required number of training samples and the expected number of function evaluations. In general, the more 


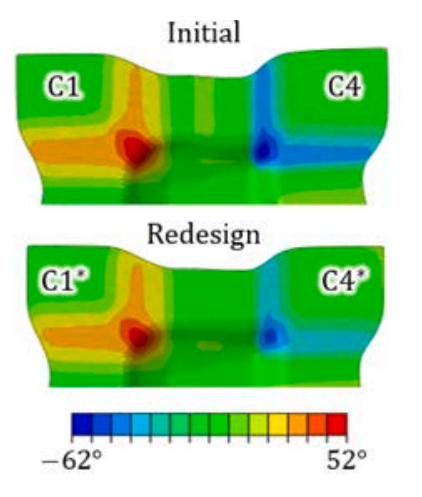

a)

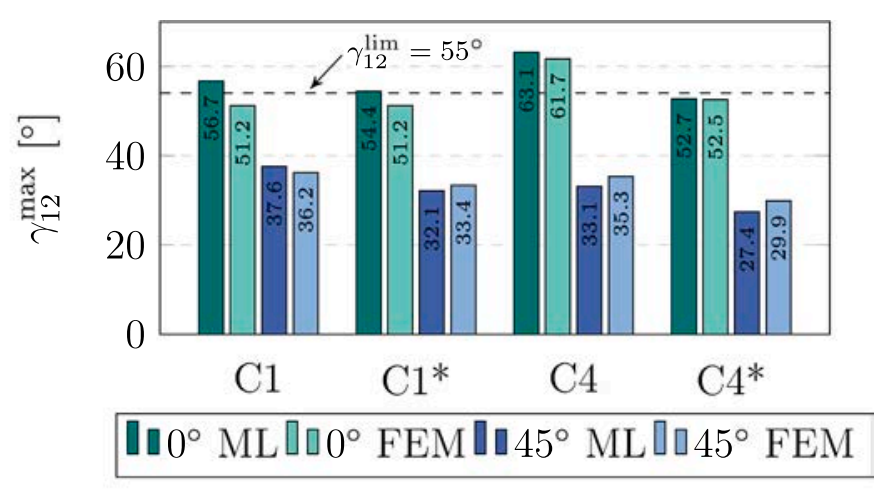

b)

Fig. 13. Visualisation of the shear angles $\gamma_{12}$ after forming for the initial corners $\mathrm{C} 1$ and $\mathrm{C} 4$ and the redesigned corners $\mathrm{C} 1 *$ and $\mathrm{C} 4 *$ (a) and a comparison of the maximum shear angle $\gamma_{12}^{\max }$ as predicted by the ML-model and an FE-simulation (b). (For interpretation of the references to colour in this figure legend, the reader is referred to the web version of this article.)

repetitive/iterative the task/optimisation is, the greater the computational benefit.

\section{Conclusion and outlook}

A machine-learning-based approach is presented to rapidly assess and improve formability of CoFRP components. To this, an algorithm identifies doubly-curved and thus potentially critical geometry features in a component of interest using an image-based recognition technique. A set of geometry parameters is extracted for each recognised feature, from which an ML-model infers individual forming results in short time. The validity of separately assessing individual features in a component is justified by Saint-Venant's Principle (SVP) and numerical verification. The approach is exemplarily applied to a complex shaped box-geometry with rounded corners. Being trained on physics-based FE-draping simulations, the ML-model is able to maintain physical accuracy while being numerically inexpensive as a use-case study shows. Additionally, design maps deduced from the ML-model give a comprehensive visualisation on the impact of local design variations. Thus, the designer can explore many design alternatives in an intuitive manner without need for laborious and computation-intensive FE-simulations.

Further investigations are still envisaged. In particular, extensions of the considered geometry range beyond a priori specified features (i.e. corners) needs to be addressed. Also, future models must be able to cope with arbitrary instead of pre-specified fibre orientations, which requires rotation invariant feature evaluation. One option to this are Convolutional Neural Networks (CNN). They are capable of learning system dynamics from generic data and can describe full-field physical effects in real-world engineering problems $[45,46]$. First results using CNNs for textile draping appear promising [47]. Also, including additional variables for, e.g. variable material properties, multiple layers or variable process conditions (e.g. temperature or tool closing profile), is of high importance in future approach developments since forming results may signficantly differ under variable conditions.

This feasibility study uses a plausible, but generic FE-fabric model for ML-approach validation. Nonetheless, being based on known mechanical theory (SVP), the developed ML-concept is deemed directly transferable to other fabric materials. Thus, in industrial applications, fully-validated material models may be used in an analogous manner for training data generation. Prospectively, such ML-approaches could result in a fast and easy-to-use software tool for engineers to evaluate and improve different part and process configurations at early stages of the design process.

\section{Acknowledgment}

The authors would like to thank the German Federal State Ministry of Science, Research and the Arts of Baden Württemberg (MWK) for funding the project "Forschungsbrücke Karlsruhe-Stuttgart", which the presented work is carried out for. Additionally, the authors would like to thank the project partners at the Institute of Aircraft Design (IFB) of Stuttgart University, lead by Prof. Peter Middendorf for fruitful collaboration and discussion. The work is also part of the Young Investigator Group (YIG) "Tailored Composite Materials for Lightweight Vehicles", generously funded by Vector Stiftung.

\section{References}

[1] Kärger L, Galkin S, Zimmerling C, Dörr D, Linden J, Oeckerath A, et al. Forming optimisation embedded in a cae chain to assess and enhance the structural performance of composite components. Compos Struct 2018;192:143-52.

[2] Kärger L, Bernath A, Fritz F, Galkin S, Magagnato D, Oeckerath A, et al. Development and validation of a CAE chain for unidirectional fibre reinforced composite components. Compos Struct 2015;132:350-8.

[3] Sharma SB, Sutcliffe M. A simplified finite element model for draping of woven material. Composites Part A 2004;35(6):637-43.

[4] Lin H, Wang J, Long AC, Clifford MJ, Harrison P. Predictive modelling for optimization of textile composite forming. Compos Sci Technol 2007;67(15-16):3242-52.

[5] Chen S, Harper LT, Endruweit A, Warrior NA. Formability optimisation of fabric preforms by controlling material draw-in through in-plane constraints. Composites Part A 2015;76:10-9.

[6] Dostaler I. Avoiding rework in product design: evidence from the aerospace industry. Int J Qual Reliab Manage 2010;27(1):5-26.

[7] Köke H, Weiß L, Hühne C, Sinapius M. A graph-based method for calculating draping strategies for the application of fiber-reinforced materials on arbitrary surfaces. Compos Struct 2017;162:123-32.

[8] Skordos AA, Sutcliffe M, Klintworth JW, Adolfsson P, editors. Multi-objective optimisation of woven composite draping unsing genetic algorithms; 2006.

[9] Dörr D, Henning F, Kärger L. Nonlinear hyperviscoelastic modelling of intra-ply deformation behaviour in finite element forming simulation of continuously fibrereinforced thermoplastics. Composites Part A 2018;109:585-96.

[10] Butenko V, Albers A. Improving the knowledge transfer from research to industry by developing demand-oriented design guidelines for fibre-reinforced plastics. Proc CIRP 2018;70:41-6.

[11] Robertson R, Chu T-J, Gerard R, Kim J-H, Park M, Kim H-G, et al. Three-dimensional fiber reinforcement shapes obtainable from flat, bidirectional fabrics without wrinkling or cutting. Part 1. A single four-sided pyramid. Composites Part A 2000;31(7):703-15.

[12] Böhler P, Härtel F, Middendorf P. Identification of forming limits for unidirectional carbon textiles in reality and mesoscopic simulation. Key Eng Mater 2013;554-557:423-32

[13] Hancock SG, Potter KD. Inverse drape modelling - an investigation of the set of shapes that can be formed from continuous aligned woven fibre reinforcements. Composites Part A 2005;36(7):947-53.

[14] Pickett AK, Creech G, de Luca P. Simplified and advanced simulation methods for prediction of fabric draping. Revue Euro Élém Finis 2005;14(6-7):677-91.

[15] Fengler B, Kärger L, Henning F, Hrymak A. Multi-objective patch optimization with integrated kinematic draping simulation for continuous-discontinuous fiber-reinforced composite structures. J Compos Sci 2018;2(2):22. 
[16] Forrester AIJ, Sóbester A, Keane AJ. Engineering design via surrogate modelling: a practical guide. Chichester: Wiley; 2008.

[17] Jansson N, Wakeman WD, Månson J-A. Optimization of hybrid thermoplastic composite structures using surrogate models and genetic algorithms. Compos Struct 2007;80(1):21-31.

[18] Arian Nik M, Fayazbakhsh K, Pasini D, Lessard L. A comparative study of metamodeling methods for the design optimization of variable stiffness composites. Compos Struct 2014;107:494-501.

[19] Dey S, Mukhopadhyay T, Adhikari S. Stochastic free vibration analyses of composite shallow doubly curved shells - a kriging model approach. Compos Part B: Eng 2015;70:99-112.

[20] Zhang Z, Friedrich K. Artificial neural networks applied to polymer composites: a review. Compos Sci Technol 2003;63(14):2029-44.

[21] El Kadi H. Modeling the mechanical behavior of fiber-reinforced polymeric composite materials using artificial neural networks-a review. Compos Struct 2006;73(1):1-23.

[22] Balokas G, Czichon S, Rolfes R. Neural network assisted multiscale analysis for the elastic properties prediction of $3 \mathrm{~d}$ braided composites under uncertainty. Compos Struct 2018;183:550-62.

[23] Heinecke F, Wille T. In-situ structural evaluation during the fibre deposition process of composite manufacturing. CEAS Aeronaut J 2018;9(1):123-33.

[24] Pfrommer J, Zimmerling C, Liu J, Kärger L, Henning F, Beyerer J. Optimisation of manufacturing process parameters using deep neural networks as surrogate models. Proc CIRP 2018;72:426-31.

[25] Zimmerling C, Pfrommer J, Liu J, Beyerer J, Henning F, Kärger L. Application and evaluation of meta-model assisted optimisation strategies for gripper-assisted fabric draping in composite manufacturing. In: Proceedings of the 18th ECCM; 2018.

[26] Zimmerling C, Dörr D, Henning F, Kärger L. A meta-model based approach for rapid formability estimation of continuous fibre reinforced components. In: AIP conference proceedings, vol. 1960; 2018. p. 020042.

[27] Simpson TW, Poplinski JD, Koch PN, Allen JK. Metamodels for computer-based engineering design: Survey and recommendations. Eng Comput 2001;17(2):129-50.

[28] Ambrogio G, Ciancio C, Filice L, Gagliardi F. Innovative metamodelling-based process design for manufacturing: an application to incremental sheet forming. Int $J$ Mater Form 2017;10(3):279-86.

[29] Kleijnen JP. Regression and kriging metamodels with their experimental designs in simulation: a review. Euro J Oper Res 2017;256(1):1-16.

[30] Fengler B, Schäferling M, Schäfer B, Bretz L, Lanza G, Häfner B, et al. Manufacturing uncertainties and resulting robustness of optimized patch positions on continuousdiscontinuous fiber reinforced polymer structures. Compos Struct 2019;213:47-57.

[31] Dey S, Mukhopadhyay T, Adhikari S. Metamodel based high-fidelity stochastic analysis of composite laminates: a concise review with critical comparative assessment. Compos Struct 2017;171:227-50.

[32] Rasmussen CE, Williams CKI. Gaussian processes for machine learning, Adaptive computation and machine learning. Cambridge Mass U.A.: MIT Press; 2006.

[33] Steger C, Ulrich M, Wiedemann C, editors. Machine vision algorithms and applications. 2nd ed.Weinheim: Wiley-VCH; 2018.

[34] Lewis JP. Fast template matching. In: Vision interface, vol. 95; 1995. p. 15-9.

[35] Ambrogio G, de Napoli L, Filice L, Muzzupappa M. Experimental evidences concerning geometrical accuracy after unclamping and trimming incrementally formed components. Key Eng Mater 2007;344:535-42.

[36] Joseph VR, Hung Y. Orthogonal-maximin latin hypercube designs. Stat Sin 2008:171-86.

[37] Bonte M, van den Boogaard AH, Huétink J. A metamodel based optimisation algorithm for metal forming processes. Advanced methods in material forming. Berlin-Heidelberg: Springer-Verlag; 2007. p. 55-72.

[38] Pazmino J, Mathieu S, Carvelli V, Boisse P, Lomov SV. Numerical modelling of forming of a non-crimp $3 \mathrm{~d}$ orthogonal weave e-glass composite reinforcement. Composites Part A 2015;72:207-18.

[39] Schirmaier F, Dörr D, Henning F, Kärger L. A macroscopic approach to simulate the forming behaviour of stitched unidirectional non-crimp fabrics [UD-NCF]. Composites Part A 2017;102:322-35.

[40] Dörr D, Schirmaier FJ, Henning F, Kärger L. A viscoelastic approach for modeling bending behavior in finite element forming simulation of continuously fiber reinforced composites. Composites Part A 2017;94:113-23.

[41] Haanappel S, ten Thije R, Sachs U, Rietman B, Akkerman R. Formability analyses of uni-directional and textile reinforced thermoplastics. Compos Part A: Appl Sci Manuf 2014;56:80-92.

[42] Guzman-Maldonado E, Hamila N, Naouar N, Moulin G, Boisse P. Simulation of thermoplastic prepreg thermoforming based on a visco-hyperelastic model a thermal homogenization. Mater Des 2016;93:431-42.

[43] Boisse P, Colmars J, Hamila N, Naouar N, Steer Q. Bending and wrinkling of composite fiber preforms and prepregs. A review and new developments in the draping simulations. Compos Part B: Eng 2018:234-49.

[44] Fung YC, Tong P, Chen X. Classical and computational solid mechanics vol. 02. World Scientific; 2017.

[45] Guo X, Li W, Iorio F. Convolutional neural networks for steady flow approximation. Proceedings of the 22nd ACM SIGKDD international conference on knowledge discovery and data mining. 2016. p. 481-90.

[46] Farimani AB, Gomes J, Pande VS. Deep learning the physics of transport phenomena. < http://arxiv.org/pdf/1709.02432v1 >

[47] Zimmerling C, Trippe D, Fengler B, Kärger L. An approach for rapid prediction of textile draping results for variable composite component geometries using deep neural networks. In: AIP conference proceedings; 2019 [accepted for publication]. 
Karlsruher Institut für Technologie

Repository KITopen

Dies ist ein Postprint/begutachtetes Manuskript.

Empfohlene Zitierung:

Zimmerling, C.; Dörr, D.; Henning, F.; Kärger, L.

A machine learning assisted approach for textile formability assessment and design improvement of composite components.

2019. Composites / A

doi: $\underline{10.5445 / I R / 1000095835}$

Zitierung der Originalveröffentlichung:

Zimmerling, C.; Dörr, D.; Henning, F.; Kärger, L.

A machine learning assisted approach for textile formability assessment and design improvement of composite components.

2019. Composites / A, 124, Art. Nr.: 105459

doi:10.1016/j.compositesa.2019.05.027

Lizenzinformationen: KITopen-Lizenz 